\title{
Effects of Branched-Chain Amino Acid and Glutamine Supplementation on Angiogenic Factors and Pro- Inflammatory Cytokines after Acute Exercise in Adolescence Athletes
}

\author{
Ju Yong Bae ${ }^{1+}$, Ga Hee Koo ${ }^{1+}$, Sung Chul Park ${ }^{2}$, Ki Ok Shin ${ }^{1 *}$ \\ ${ }^{1}$ Laboratory of Exercise Biochemistry, Department of Physical Education, Dong-A University, Busan, Korea \\ ${ }^{2}$ Department of Rehabilitation Exercise \& Health, Busan Institute of Science and Techology \\ + Both authors contributed equally to this work
}

Received: February 18, 2019

Accepted: April 24, 2019

Published online: April 30, 2019

Keywords:

Ang-1

BCAA

FLRG

Glutamine

VEGF

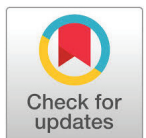

\section{ABSTRACT}

OBJECTIVES The purpose of this study was to investigate the changes in the concentration of blood angiogenic factors and pro-inflammatory cytokines during acute exercise after the administration of branched-chain amino acid (BCAA) and glutamine.

METHODS All 7 adolescent male athletes performed $2000 \mathrm{~m}$ rowing exercise after the completion of each of three different 7-day treatments; non-treatment (CO); a total daily dose of BCAA $3.15 \mathrm{~g}(1.05 \mathrm{~g} \times 3)$ (BCAA); or both a total daily dose of BCAA $3.15 \mathrm{~g}(1.05 \mathrm{~g} \times 3)$ and glutamine $6 \mathrm{~g}(2 \mathrm{~g} \times 3)(\mathrm{BC}+\mathrm{GLU})$. There was a 30-day rest period between each treatment. After intake of amino acids for 7 days, the participants completed the rowing exercise on day 8 , and their blood samples were collected (at resting state, Rest; immediately after exercise, Ex0; and $30 \mathrm{~min}$ after the exercise, Ex30) and were analyzed using ELISA.

RESULTS The concentration of Vascular endothelial growth factor (VEGF) increased in the BCAA and BC+GLU treatment groups compared to that at Rest in the CO group $(p<0.05)$. Interleukin- 6 (IL-6) increased at Ex0 compared to that at Rest in the $\mathrm{CO}$ and BCAA treatment groups $(p<0.05)$, and significantly decreased at Ex30 compared to that at ExO in the BCAA treatment group $(p<0.05)$. However, significant differences were not observed in $\mathrm{BC}+\mathrm{GLU}$ at Ex0 and Ex30 compared to at Rest. IL-8 also significantly increased at Ex0 compared to at Rest, and significantly decreased at Ex30 compared to at Ex0 in $\mathrm{CO}(\mathrm{p}<0.05)$. However, the blood IL-8 level in BCAA and BC+GLU were not altered by acute exercise.

CONCLUSIONS This study suggested that the administrations of BCAA and/or glutamine have enough to induce increase of VEGF concentration and inhibited the increase of pro-inflammatory cytokines after acute exercise in adolescence athletes.

๑ The Asian Society of Kinesiology and the Korean Academy of Kinesiology

\section{Introduction}

Branched-chain amino acids (BCAA) account for approximately $40 \%$ of the essential amino acids in blood and

*Correspondence: Ki Ok Shin, Department of Physical Education, College of Sports Sciences, Dong-A University, Hadan 2 dong, Saha-gu, Busan, 604-714, Republic of Korea; Tel: +82-51-200-7808; Fax:+82-51-200-7805; E-mail: kshin21@dau.ac.kr play important roles in skeletal muscle growth [1]. BCAA are important precursors of tricarboxylic acid (TCA) cycle intermediates via acetyl-CoA and Succinyl-CoA and can be involved in energy production through the modulation of exercise-induced serum BCAAs oxidation [2,3]. Glutamine is the most abundant protein in the blood $(\sim 600-700 \mu \mathrm{M})$ 
and accounts for approximately $60 \%$ of the free amino acids in the muscle [4]. In addition, it enhances protein synthesis by increasing the size of the muscle cells [5]. The study of BCAAs and glutamine supplementation has been focused on a potential nutritional strategy to avoid or alleviate exerciseinduced muscle damage [6].

Angiogenic factors are known to be secreted from contracting skeletal muscles and play positive roles in muscle growth and muscle hypertrophy by stimulating angiogenesis [7]. Typical signaling proteins of angiogenesis include vascular endothelial growth factor (VEGF), angiopoeitin-1 (Ang-1), and follistatin (FLRG) [8,9]. VEGF plays an essential role in increasing vessel density of the skeletal muscle induced by exercise and maintaining of the supply of muscle capillary vessels in the skeletal muscle [10]. Ang-1 has been known to prevent the apoptosis of endothelial cells and is associated with vascular stabilization [11]. In addition, FLRG, which is an antagonist of myostatin that causes muscle wasting, has been reported to play an important role in the regulation of myostatin activity in vivo [11].

Previous studies have reported that amino acid supplementation for muscle hypertrophy not only increases anabolism in muscle tissues and facilitates recovery after training by supplying essential amino acids, but also positively affects protein synthesis to a greater extent than dietary reference intake [12]. However, other studies have reported that BCAA supplementation possibly generates energy depletion [13] by reducing a-ketoglutarate, an intermediate of Krebs Cycle [14]. Therefore, not only the effect of BCAA supplementation but also the effect of BCAA and glutamine combined treatment is unclear.

Acute exercise causes an inflammatory response characterized by transient elevation of pro-inflammatory cytokines such as IL-6, and IL-8 $[15,16]$. The increase of cytokines to acute exercise are dependent on the type and intensity of exercise, especially, higher pro-inflammatory cytokine levels are observed after an exhaustive acute exercise bout such as marathon [15] and triathlon [17] competition. Especially, in the presence of consecutive matches, it is considered essential to control inflammatory cytokines in conjunction with muscle injury in terms of recovery after acute exercise.

Accordingly, this study was conducted in male adolescent athletes to observe the changes in concentrations of blood angiogenic factors and pro-inflammatory cytokines during acute exercise after administration of BCAA and glutamine.

\section{Materials and methods}

\section{Subjects}

Seven male adolescent yacht athletes (age: 17.7 \pm 0.74 years; height: $175.3 \pm 1.38 \mathrm{~cm}$; weight: $67.5 \pm 4.87 \mathrm{~kg}$; BMI: $21.9 \pm 1.40 \mathrm{~kg} / \mathrm{m}^{2}$; fat: $15.6 \pm 2.34 \%$; lean body mass: $56.3 \pm 2.54$ kg; career: 3.57 years) participated in this study. The subjects could carry out low intensity exercise to maintain physical fitness, which had no effect on muscle mass and body fat mass during whole experimental periods, because this study was conducted in the off-season.

All subjects were given detailed explanations of the experimental procedures before the start of the experiment, and they submitted an agreement for the experiment including that they could freely withdraw from the experiment at any time if they did not want it. To obtain reliable results in the study, the subjects were asked not to conduct excessive physical activities on the day of the study. Subjects were not allowed to take drugs or dietary therapy. In addition, the subjects were in a 12-hour fasting state on the day of the study.

\section{Experimental procedures}

Each of the 7 participants completed all the three experiments, with a 1 month resting period between each experiment. Non-supplemented treatment (CO), BCAA-

Table 1. Changes of body composition after each treatment $(n=7)$

\begin{tabular}{cccc}
\hline & CO & BCAA & BC+GLU \\
\hline Weight $(\mathrm{kg})$ & $67.54 \pm 4.87$ & $68.38 \pm 4.76$ & $68.10 \pm 4.54$ \\
BMI $\left(\mathrm{kg} / \mathrm{m}^{2}\right)$ & $21.90 \pm 1.40$ & $22.17 \pm 1.36$ & $22.09 \pm 1.31$ \\
$\%$ Body fat (\%) & $15.6 \pm 2.34$ & $15.77 \pm 2.17$ & $17.13 \pm 5.96$ \\
LBM $(\mathrm{kg})$ & $56.33 \pm 2.54$ & $57.01 \pm 2.66$ & $55.83 \pm 2.31$ \\
\hline
\end{tabular}

Values are presented as mean \pm SE. CO; Non supplements treatment, BCAA; BCAA supplements treatment, $B C+G L U ; B C A A$ and Glutamine supplements treatment, $B M l$; body mass index, LBM; lean body mass. 


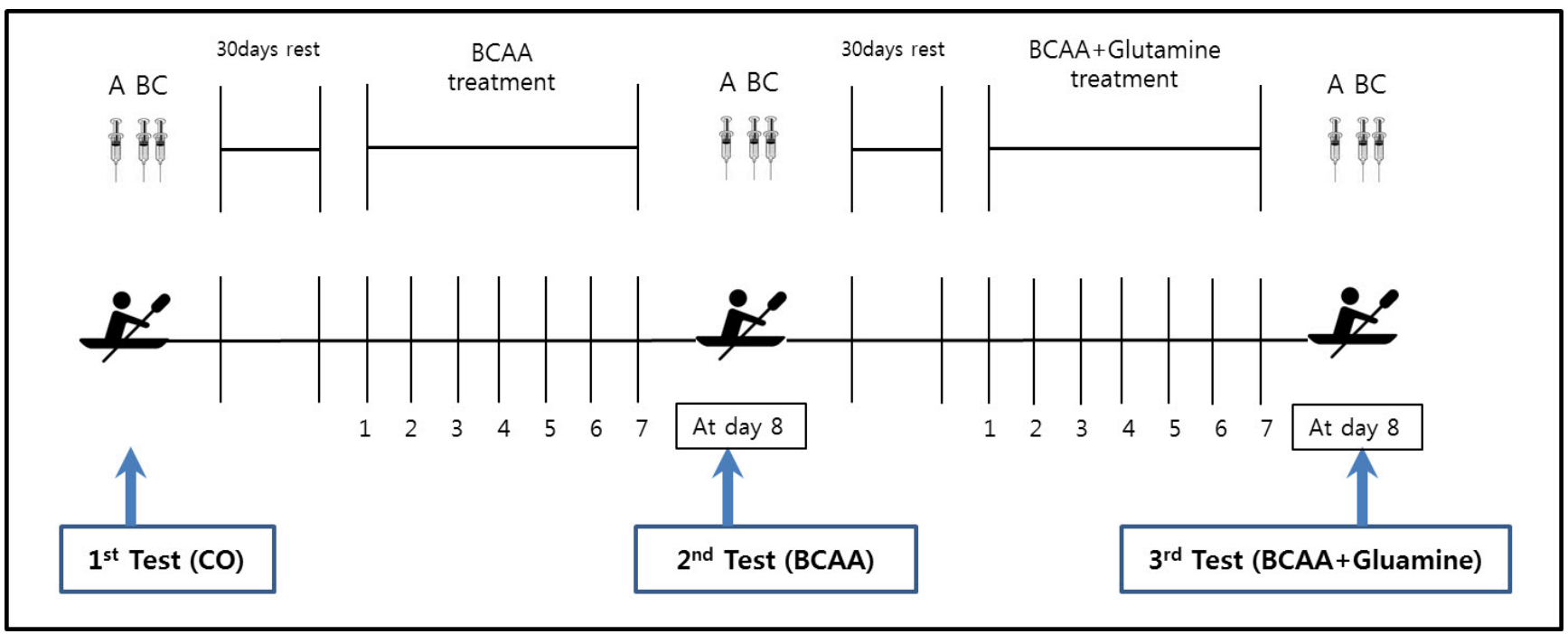

Figure 1. Experimental design

supplemented treatment (valine: $25 \%$, leucine: $50 \%$, isoleucine: $25 \%, 3.15 \mathrm{~g} /$ day) in a capsule (BCAA), and BCAA- and glutamine-supplemented treatment (L-glutamine, Optimum Nutrition Co., 6 g/day) (BC + GLU) were administered to the subjects three times per day (after breakfast, lunch, and dinner) for 7 days. On day 8 , an acute $2000 \mathrm{~m}$ rowing exercise was conducted. Blood samples were collected three times for each experiment: the first blood sample was collected at resting state before the rowing exercise (Rest); the second sample was collected immediately after conducting the rowing exercise (Ex0); and the last blood sample was collected at 30 minutes after the rowing exercise (Ex30).

7 yacht athletes participated and were each completed three experiments (CO, BCAA, BC+GLU). Blood samples were collected three times for each experiment $(A, B, C)$. CO; Non supplements treatment, BCAA; BCAA supplements treatment, $\mathrm{BC}+\mathrm{GLU}$; $\mathrm{BCAA}$ and Glutamine supplements treatment. A; Blood sampling at rest status before exercise (Rest), B; Blood sampling at immediately after exercise (Ex0), C; Blood sampling at 30 minutes after Ex0 (Ex30).

\section{$2000 \mathrm{~m}$ rowing exercise}

After 7 days of each amino acid administration, a graded exercise test was conducted using a Concept2 Indoor Rower (USA) before execution of the $2000 \mathrm{~m}$ rowing exercise. For the determination of exercise intensity, the Standard List 2000 $\mathrm{m}$ protocol that is set using the Select Workout function of Concept 2 Indoor Rower was selected and the subjects were asked to complete the protocol with their maximum effort.

\section{Analysis of blood samples}

A blood sample of $10 \mathrm{cc}$ was collected from the forearm vein of the subjects. The samples were centrifuged at 3,000 rpm for $10 \mathrm{~min}$, followed by storage at $80^{\circ} \mathrm{C}$ until analysis.

\section{Angiogenic factors}

VEGF, Ang-1, and FLRG were analyzed using a R\&D system kit. The capture antibody $(100 \mu \mathrm{l})$ was incubated overnight and washed three times. Then, the sample and standard were added, followed by a 2 -hour incubation at room temperature to prepare for the aliquot of the detection antibody. Thereafter, streptavidin-HRP and substrate solution were combined for a color reaction, and then stop solution was added to the reaction $20 \mathrm{~min}$ later, followed by measurement of absorbance at $450 \mathrm{~nm}$.

\section{Pro-inflammatory cytokines}

Biochemical analyses of IL- 6 and IL-8 were conducted using a human serum cytokine kit via a Bio-Plex pro magnetic cytokine assay. Standards, samples, and cytokine magnetic beads were diluted respectively. A $50 \mu$ laliquot of each the diluted standard and sample, respectively, was put into a well, 
followed by 30 -min shaker incubation, and then washing three times. Next, the detection antibody was prepared and a $50 \mu \mathrm{l}$ aliquot was added to each well and incubated on a shaker for 30-min, followed by three washes. StreptavidinPE was prepared using assay buffer, and a $50 \mu$ laliquot of streptavidin-PE was added to each well, followed by a 10-min shaker incubation, and then washing three times. A $125 \mu \mathrm{l}$ aliquot of assay buffer was added to each well, followed by $30 \mathrm{~s}$ shaking. Afterwards, the mixture was analyzed using Bio-Plex computer Manager (Bio-Rad).

\section{Statistical analyses}

Data obtained from this study was analyzed using SPSS Window Version 18.0. The mean and standard deviation of the levels of blood angiogenic factors and pro-inflammatory cytokines that had been changed were calculated in each state. Two-way repeated measures ANOVA was conducted to investigate differences in the levels between ergogenic aid and time course. One-way ANOVA was conducted to investigate differences in the levels over time of each treatment. A back-testing was conducted using Duncan for significance validation. A significant level of $\alpha=0.05$ was set for statistical analysis.

\section{Results}

Changes in blood VEGF levels by amino acid administration are presented in Fig 2A. Significant differences were observed with the $\mathrm{CO}$ at Ex0 and Ex30 compared to at Rest $(p<0.05)$ and was also observed between Ex30 and Ex0 $(\mathrm{p}<0.05)$. In the comparison among treatments, the blood VEGF level at Rest was shown to have significantly increased in the BCAA and $\mathrm{BC}+\mathrm{GLU}$ compared to the $\mathrm{CO}(\mathrm{p}<0.05)$. However, no changes in blood Ang-1 and FLRG levels were observed.

Mean \pm SE, CO; Non supplements treatment, BCAA; BCAA supplements treatment, $\mathrm{BC}+\mathrm{GLU}$; BCAA and Glutamine supplements treatment, Rest; Blood sampling at rest status before exercise, Ex0; Blood sampling at immediately after exercise, Ex30; Blood sampling at 30 minutes after Ex0. ${ }^{*} \mathrm{p}<.05$; Significant difference compared with Rest, ${ }^{\ddagger} \mathrm{p}<.05$; Significant difference compared with Ex $0, \# p<.05$; Significant difference
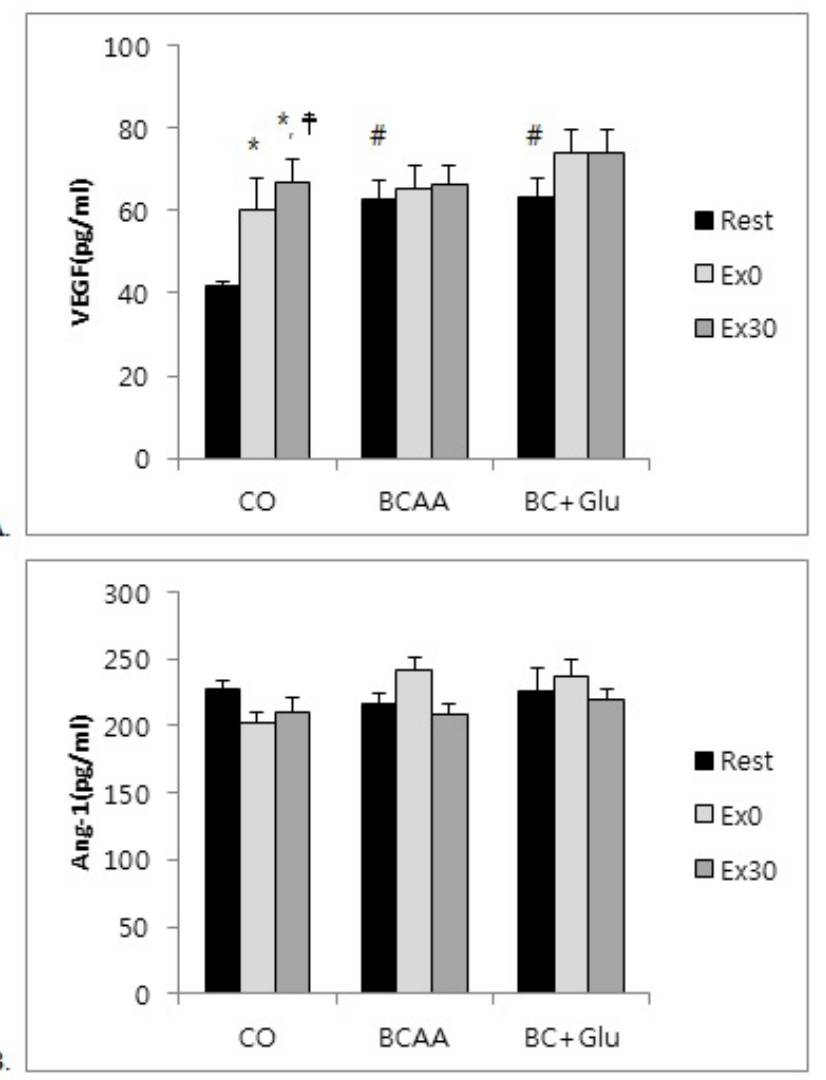

B.

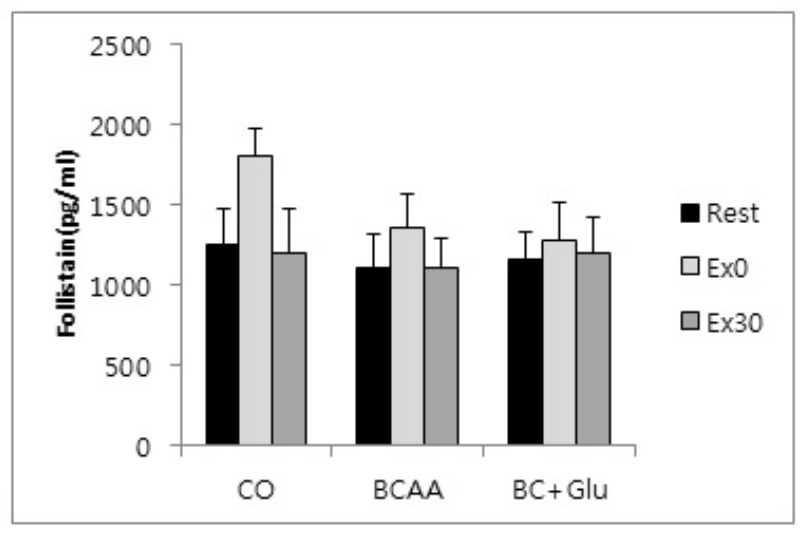

Figure 2. Changes of plasma angiogenesis factors according to the amino acid supplementation

compared with Rest in CO.

Mean \pm SE, CO; Non supplements treatment, BCAA; BCAA supplements treatment, $\mathrm{BC}+\mathrm{GLU}$; BCAA and Glutamine supplements treatment, Rest; Blood sampling at rest status before exercise, Ex0; Blood sampling at immediately after exercise, Ex30; Blood sampling at 30 minutes after Ex0. ${ }^{*} \mathrm{p}<.05$; Significant difference compared with Rest, ${ }^{\ddagger} \mathrm{p}<.05$; Significant difference compared with Ex0. 


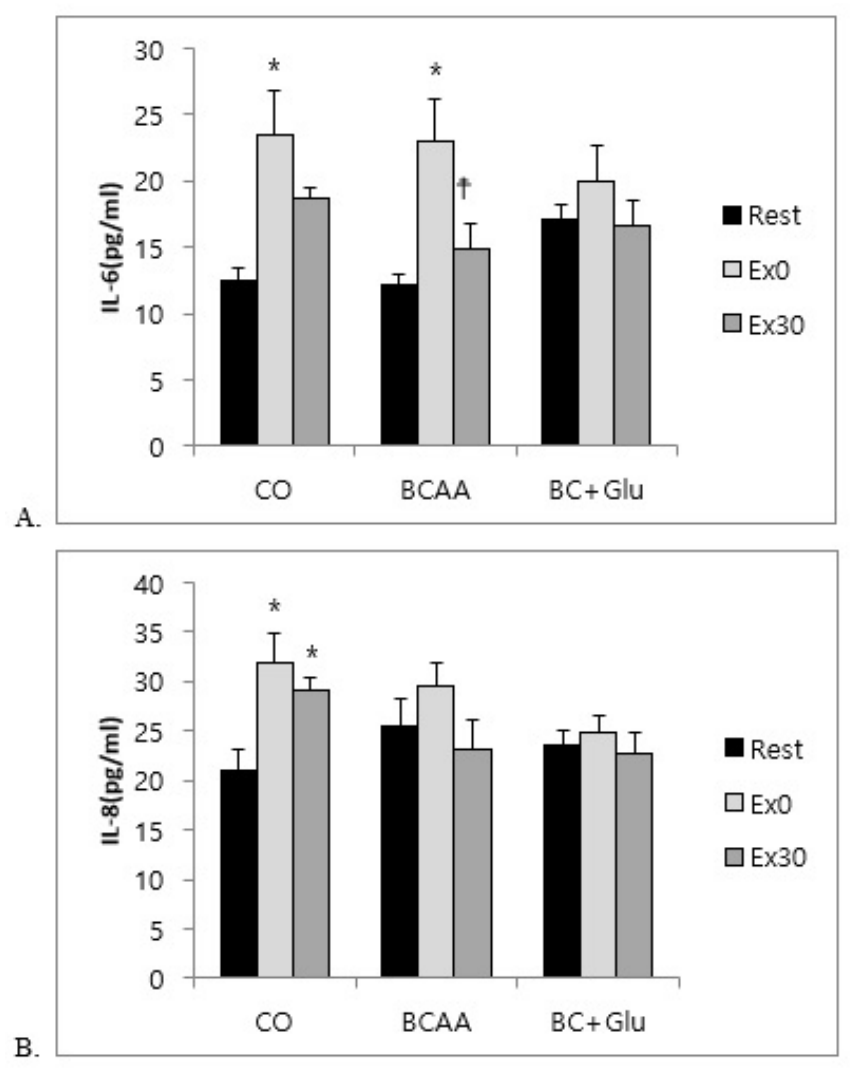

Figure 3. Changes of plasma pro-inflammatory cytokines according to the amino acid supplementation

As shown in Fig 3A, for changes in the blood IL-6 level by amino acid administration, the level is shown to have significantly increased with $\mathrm{CO}$ and BCAA treatments at Ex0 compared to at Rest $(\mathrm{p}<0.05)$, but it was shown to have significantly decreased with BCAA at Ex30 compared to at Ex0 $(\mathrm{p}<0.05)$. However, significant differences were not observed with $\mathrm{BC}+\mathrm{GLU}$ at Ex0 and Ex30 compared to at Rest.

As shown in Fig 3B, for changes in the blood IL-8 level, the level was shown to have significantly increased at Ex0 and Ex30 in CO compared to at Rest $(\mathrm{p}<0.05)$. However, the blood IL- 8 level in BCAA and BC+GLU were not altered by acute exercise.

\section{Discussion}

Intake of amino acid supplements makes directly/ indirectly improving training effect and performance, result from utilization of energy sources. Therefore, this study focused on the effect of acute exercise on angiogenesis and pro-inflammatory cytokines after consumption of BCAA and glutamine supplements.

Angiogenesis is a known process in the formation of new blood vessels and increases in capillary vessels, and the pathways of angiogenesis are divided into signaling mediated by either VEGF or angiopoietin (Ang) [8]. VEGF increases the diffusion of endothelial cells and cell movement [18], and Ang affects vascular stabilization [19]. Notably, Ang-1 is expressed by the stimulation of VEGF, playing an important role in vascular maturation and stabilization [20]. FLRG, another angiogenic factor, is a constituent of the transforming growth factor beta (TGF- $\beta$ ) superfamily, and is known to inhibit the activity of myostatin, which regulates skeletal muscle growth [9]. Myostatin neutralization caused by follistatin significantly affects skeletal muscle growth, and the overexpression of follistatin promotes muscle hypertrophy by activating satellite cells [21]. In general, both aerobic exercise and resistant exercise have been known to promote angiogenesis in skeletal muscle [22,23].

Considering that VEGF levels increase most rapidly within $30 \mathrm{~min}$ after exercise, skeletal muscle responses to exercise are very sensitive [23]. In this study, the blood VEGF level after acute rowing exercise was significantly increased immediately and at 30 min after the exercise in CO, consistent with the previous study [24]. In case of BCAA and BC+GLU treatments, the significant increases in blood VEGF levels were confirmed at the rest compared to that of the control group, and this result indicate that supplementation for 7 days is effective enough to increase VEGF levels at rest status. However, the blood VEGF level immediately after exercise was not shown in the BCAA and BC+GLU, and this was probably due to the sufficiently increased at the rest status.

However, increases in blood Ang-1 and FLRG levels were not induced after consumption of amino acids and exercise in this study. Increases in Ang-1 after conducting of eccentric exercise were negatively affected by poor conditions of basal membrane and pericytes, and tendon injury [25]. The aforementioned results were reported to be attributable to the fact that a longer exercise duration is required for smooth blood circulation in the tendon [20]. No significant difference in Ang-1 levels observed in this study is likely to be attributable 
to the acute insufficiency of the blood flow caused by intensive exercise, as shown in the previous study. In addition, Mero et al. reported that blood amino acid levels at resting state significantly increased with the administration of $6 \mathrm{~g}$ BCAA (4 g leucine, $1 \mathrm{~g}$ isoleucine, $1 \mathrm{~g}$ valine) [26]. Previous studies have reported that BCAA supplementation $>5 \mathrm{~g} \cdot \mathrm{kg}^{-1} \mathrm{~d}^{-1}$ should be recommended with a regard to exercise [27]. However, Tarnopolsky reported that an intake of protein $\leq 1.0 \mathrm{~g} \cdot \mathrm{kg}^{-1} \cdot \mathrm{d}^{-1}$ was insufficient for protein metabolism required for exercise in both male and female athletes [28]. Thus, no significant change observed in this study is likely to be attributable to the relatively lower dose than the recommended dose in the previous study. Therefore, a further study on the effect of sufficient administration of BCAA and glutamine on angiogenic factors is required.

Many studies have reported that both acute and regular exercise induce increases in circulating cytokines level. Endurance exercise induced acute inflammatory response in athletes [29] that may lead to chronic inflammation status in case of further extensive training session [30]. In addition, single prolonged exercise can induce acute elevations in pro-inflammatory cytokines such as IL-6, IL-8 in endurance athletes [31]. IL-6 level significantly increased after highintensity knee extension exercise [32], and IL-8 level was reported to increase after exhaustive exercise consisting of concentric and eccentric exercises [33]. On the other hand, no changes in blood cytokine levels were detected after 1-hour of rowing [34] or after 2-hours of cycling [35]. As mentioned above, the training effects are not clear and are affected by various factors, such as exercise intensity, exercise duration, and muscle mass used during exercise [36]. In this study, acute exercise increased levels of pro-inflammatory cytokines, but 7 days of $\mathrm{BC}+\mathrm{GLU}$ treatment inhibited the increase of circulating IL-6 level. Moreover, treatment of BCAA also showed a rapid increase of IL-6 immediately after exercise, but the level was reduced similarly to the rest state. The circulating IL-8 level also increased after acute exercise, but 7 days of BCAA and BC + GLU treatments inhibited the increase of circulating IL-8 level. These results suggested that BCAA and $\mathrm{BC}+\mathrm{GLU}$ treatments have the positive effect on regulation of pro-inflammatory cytokines after acute exercise.

\section{Conclusions}

This study suggested that the administrations of BCAA and/or glutamine have enough to induce increase of VEGF concentration and inhibited the increase of pro-inflammatory cytokines after acute exercise in adolescence athletes.

\section{Acknowledgments:}

This study was supported by research funds from Dong-A University (Research year achievement).

\section{Conflicts of Interest:}

The authors declare no conflict of interest.

\section{References}

1. Kitsy A, Carney S, Vivar JC, Knight MS, Pointer MA, Gwathmey JK, et al. Effects of leucine supplementation and serum withdrawal on branched-chain amino acid pathway gene and protein expression in mouse adipocytes. PLoS One. 2014; 22:e102615.

2. Valerio A, D’Antona G, Nisoli E. Branched-chain amino acids, mitochondrial biogenesis, and healthspan: an evolutionary perspective. Aging (Albany NY). 2011; 3(5):464-78.

3. Shimomura Y, Kobayashi H, Mawatari K, Akita K, Inaguma A, Watanabe S, et al. Effects of squat exercise and branched-chain amino acid supplementation on plasma free amino acid concentrations in young women. J Nutr Sci Vitaminol (Tokyo). 2009; 55(3):288-91.

4. Calder PC. Glutamine and the immune system. Clin Nutr. $1994 ; 13(1): 2-8$.

5. Smith RJ, Wilmore DW. Glutamine nutrition and requirements. J Parenter Enteral Nutr.1990;14(4): 94S-99S.

6. Fouré A, Bendahan D. Is branched-chain amino acids supplementation an efficient nutritional strategy to alleviate skeletal muscle damage? A systematic review. Nutrients. 2017;21;9(10).

7. Yeo NH, Woo J, Shin KO, Park JY, Kang S. The effects of 
different exercise intensity on myokine and angiogenesis factors. J Sports Med Phys Fitness. 2012; 52(4):448-454.

8. Papetti M, Herman IM. Mechanisms of normal and tumorderived angiognensis. Am J Physiol Cell Physiol. 2002; 282:C947-970.

9. Phillips DJ, de Kretser DM. Follistatin: a multifunctional regulatory protein. Front Neuroendocrinol. 1998; 19:287-322.

10. Kraus RM, Stallings HW, Yeager RC, Gavin TP. Circulating plasma VEGF response to exercise in sedentary and endurance-trained men. J Appl Physiol. 2003; 96:14451450.

11. Lee SJ. Regulation of muscle mass by myostatin. Annu Rev Cell Dev Biol. 2004; 20:61-86.

12. Koba T, Hamada K, Sakurai M. Branched-chain amino acids supplementation attenuates the accumulation of blood lactate dehydrogenase during distance running. J Sports Med Phys Fitness. 2007; 47:316-322.

13. Wagenmakers AJ, Coakley JH, Edwards RH. Metabolism of branched-chain amino acids and ammonia during exercise: clues from McArdle's disease. Int J Sports Med. 1990; 11:S101-113.

14. Van Hall G, MacLean DA, Saltin B, Wagenmakers AJ. Mechanisms of activation of muscle branched-chain alpha-keto acid dehydrogenase during exercise in man. J Physiol. 1996; 494:899-905.

15. Suzuki K, Nakaji S, Yamada M, Totsuka M, Sato K, Sugawara K. Systemic inflammatory response to exhaustive exercise. Cytokine kinetics. Exerc Immunol Rev. 2002; 8:6-48.

16. Rhodus NL, Cheng B, Myers S, Bowles W, Ho V, Ondrey F. A comparison of the pro-inflammatory, NF-kappaBdependent cytokines: TNF-alpha, IL-1-alpha, IL-6, and IL- 8 in different oral fluids from oral lichen planus patients. Clin Immunol. 2005; 114(3):278-83.

17. Huang WC, Wei CC2, Huang CC3, Chen WL4, Huang HY. The beneficial effects of lactobacillus plantarum ps128 on high-intensity, exercise-induced oxidative stress, inflammation, and performance in triathletes. Nutrients. 2019; 7;11(2).

18. Connolly DT, Heuvelman DM, Nelson R, et al. Tumor vascular permeability factor stimulates endothelial cell growth and angiogenesis. J Clin Invest. 1989; 84:14701478.

19. Waltenberger J, Claesson-Welsh L, Siegbahn A, Shibuya M, Heldin CH. Different signal transduction properties of KDR and Flt1, two receptors for vascular endothelial growth factor. J Biol Chem. 1994; 269:26988-26995.

20. Nakamura K, Kitaoka K, Tomita K. Effect of eccentric exercise on the healing process of injured patellar tendon in rats. J Orthop Sci. 2008; 13:371-378.

21. Gilson H, Schakman O, Kalista S, et al. Follistatin induces muscle hypertrophy through satellite cell proliferation and inhibition of both myostatin and activin. Am J Physiol Endocrinol Metab. 2009; 297:E157-164.

22. Wagner PD, Olfert IM, Tang K, Breen EC. Muscle-targeted deletion of VEGF and exercise capacity in mice. Respir Physiol Neurobiol. 2006; 151:159-166.

23. Green H, Goreham C, Ouyang J, Ball-Burnett M, Ranney D. Regulation of fiber size, oxidative potential, and capillarization in human muscle by resistance exercise. Am J Physiol. 1999; 276:R591-596.

24. Gustafsson T, Puntschart A, Kaifser L, Jansson E, Sundberg CJ. Exercise-induced expression of angiogenesis-related transcription and growth factors in human skeletal muscle. Am J Physiol. 1999; 276:H679-685.

25. Gavin TP, Wagner PD. Effect of short-term exercise training on angiogenic growth factor gene responses in rats. J Appl Physiol. 2001; 90:1219-1226.

26. Mero A, Leikas A, Knuutinen J, Hulmi JJ, Kovanen V. Effect of strength training session on plasma amino acid concentration following oral ingestion of leucine, BCAAs or glutamine in men. Eur J Appl Physiol. 2009; 105:215-223.

27. Shimomura Y, Murakami T, Nakai N, Nagasaki M, Harris RA. Exercise promotes BCAA catabolism: effects of BCAA supplementation on skeletal muscle during exercise. J Nutr. 2004; 134:1583-1587.

28. Tarnopolsky M. Protein requirements for endurance athletes. Nutrition. 2004; 20:662-668.

29. Nieman DC, Konrad M, Henson DA, Kennerly K, Shanely RA, Wallner-Liebmann SJ. Variance in the acute 
inflammatory response to prolonged cycling is linked to exercise intensity. J Interferon Cytokine Res 2012; 32:12-17.

30. Jürimäe J, Mäestu J, Jürimäe T, Mangus B, von Duvillard SP. Peripheral signals of energy homeostasis as possible markers of training stress in athletes: a review. Metab Clin Exp 2011; 60:335-350.

31. Comassi M, Vitolo E, Pratali L, Del Turco S, Dellanoce C, Rossi C, et al. Acute effects of different degrees of ultra-endurance exercise on systemic inflammatory responses. Intern Med J 2015; 45:74-79.

32. Helge JW, Stallknecht B, Pedersen BK, et al. The effect of graded exercise on IL- 6 realease and glucose uptake in human skeletal muscle. J Physiol. 2003; 546:299-305.

33. Suzuki K, Nakaji S, Yamada M, et al. Impact of a competitive marathon race on systemic cytokine and neutrophil responses. Med Sci Sports Exerc. 2003; 35:348-355.

34. Henson DA, Nieman DC, Nehlsen-Cannarella SL, et al. Influence of carbohydrate on cytokine and phagocytic responses to $2 \mathrm{~h}$ of rowing. Med Sci Sports Exerc. 2000; 32:1384-1389.

35. Chan MH, Carey AL, Watt MJ, Febbraio MA. Cytokine gene expression in human skeletal muscle during concentric contraction: evidence that IL-8, like IL-6, is influenced by glycogen availability. Am J Physiol Regul Integr Comp Physiol. 2004; 287:R322-327.

36. Febbraio MA, Pedersen BK. Muscle-derived interleukin-6: mechanisms for activation and possible biological roles. FASEB J. 2002; 16:1335-1347. 